\title{
Failure Analysis of Hub Bolt of Main Landing Gearing
}

\author{
Yuan Guo-he ${ }^{1}$, Jao Yang ${ }^{2}$, Wang Chun ${ }^{2}$, Feng Yan-peng, ${ }^{2, *}$ \\ ${ }^{1}$ KLK Jiangsu Cobalt Nicket Metal Co., Ltd., Jiangsu Taixing 225404, China; \\ ${ }^{2}$ China academy of civil aviation science and technology, Beijing 100028, China. \\ *fengyp@mail.castc.org.cn
}

Keywords: Hub bolt; Cadmium plating; Hydrogen embrittlement.

\begin{abstract}
The abstract should summarize the contents of the paper and should contain at least 100 and at most 300 words. It should be set in 12-point font size. There should be a space before of 18-point and after of 60-point.Hub bolt is one of the critical components to guarantee the safety during the approach and takeoff process of aircraft. One hub bolt was found broken in middle side after several hundred hours' service. Optical microscope and scanning electron microscope were used to investigate the macro- and micro-structures of broken bolt and fracture. That was fatigue characteristic, intergranular fracture with claw pattern was found near the propagation region, and a corrosion pit was found on the side of fatigue source. Cooperation with area-scan function of Energy Distribution Spectrum(EDS), it shown that the bolt on cadmium plated treatment, process condition is not appropriate. Raw cadmium plating bulge and broken in the formation of the pit, during subsequent passivation, amounts of chromium deposit on the pit position and closed depressions, while, NDT cannot effectively detect defects before installed.
\end{abstract}

\section{Introduction}

Main wheel hub of transport aircraft is a component to bearing large load, while hub bolts are critical components to fix tires and guarantee the safety during the approach and takeoff process. The cadmium plated bolt technology is used to prevent chemical corrosion of component. The hydrogen embrittlement fracture caused by cadmium plating is a typical failure mode of high strength bolts ${ }^{[1][2]}$. Due to the delay and concealment characteristics of hydrogen embrittlement fracture, it can't be detected by general non-destructive testing. So the bolt damage caused by hydrogen embrittlement fracture is much greater than that caused by the other fracture ${ }^{[3][4]}$.

Hydrogen embrittlement micro-mechanism of the research, "Cottrell atmosphere" theory has made outstanding contributions for understanding of the mechanism of hydrogen embrittlement and engineering control. However, the hydrogen embrittlement problem is still occurring due to the complexity of the electroplating and coating process in the practical engineering field, especially for the high strength steel treated by plating ${ }^{[5]}$. Xufen shao studied cadmium plating of $300 \mathrm{M}$ steel fatigue properties of hardness, which demonstrates that the fatigue crack mainly originated in the 
interface layer of cadmium plating or coating and substrate and the fatigue properties of cadmium decreased mainly related with the characteristics of cadmium plating ${ }^{[6]}$. Xu Shiqing and other research shows that in the removal of hydrogen completely, hydrogen to three dimensional stress diffusion and agglomeration in the process of using, the formation of micro cracks, hydrogen continuously to the micro crack tip extension and enrichment, many micro cracks formed and connected become macro cracks and produce hydrogen embrittlement ${ }^{[7]}$.

There are a lot of new phenomena and new features ${ }^{[1]}$ in hydrogen embrittlement fracture with the gradually improve of understanding of hydrogen embrittlement process and application of highstrength structural materials. of the. Due to the hidden character of hydrogen embrittlement damage gradually hidden, it is difficult to detect by ordinary NDT in some cases.

A company in the process of sending the aircraft, after the launch of maintenance personnel to return to the stop position, a broken bolt was found in the vicinity of the parking spaces. The new hub bolt through the relevant NDT was found broken in middle side after only several hundred hours' service. In order to avoid similar failures optical microscope and scanning electron microscope were used to investigate the macro- and micro-structures of broken bolt and fracture.

\section{Test procedure and results}

\subsection{Macroscopic morphology}

Bolt mounting position is shown in Figure 1a. There are not structure parts or other parts that occur collision or friction with the bolt. The macro morphology of the fracture bolt is shown in Figure $1 \mathrm{~b}$. The fracture position is located at the middle end of the screw bolt. Hub bolts stress state is tensile stress, usually the root of the bolt is stress concentration area, while the load distribution in middle of the bolt rod is relatively uniform.

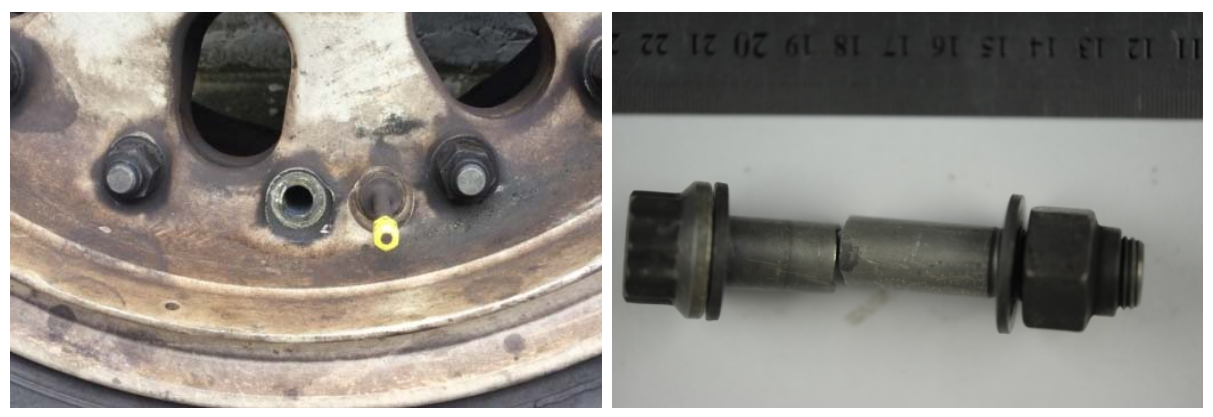

Figure 1: Macro appearance of installation site and broken bolt

\subsection{Micro topography analysis}

\subsubsection{Fracture microstructure}

The microstructure of the fracture surface of the bolt is shown in Fig. 2. The fracture surface is characterized by fatigue fracture. The boundaries of the extension are shown by the dashed blue line in Figure 2a. The direction of crack propagation is shown by the arrows in the figure. The micromorphology of the crack source region is shown in Fig. 2c, which is characterized by point source. The origin of the crack is located in the concave of the surface, and there are obvious cracks in the vicinity. 
The microcosmic morphology of the crack propagation region is shown in Fig. 3, and the microscopic morphology is intergranular cracking, and the surface of the crack is characterized by "claw pattern". A large number of secondary cracks were observed in the vicinity of the crack source, and the cracks were as shown in Fig. 3b. The Energy Distribution Spectrum of the crack propagation region shows that the metal cadmium content is abnormal. Cadmium is usually used as a protective coating and the matrix does not contain such a large amount of $\mathrm{Cd}$.
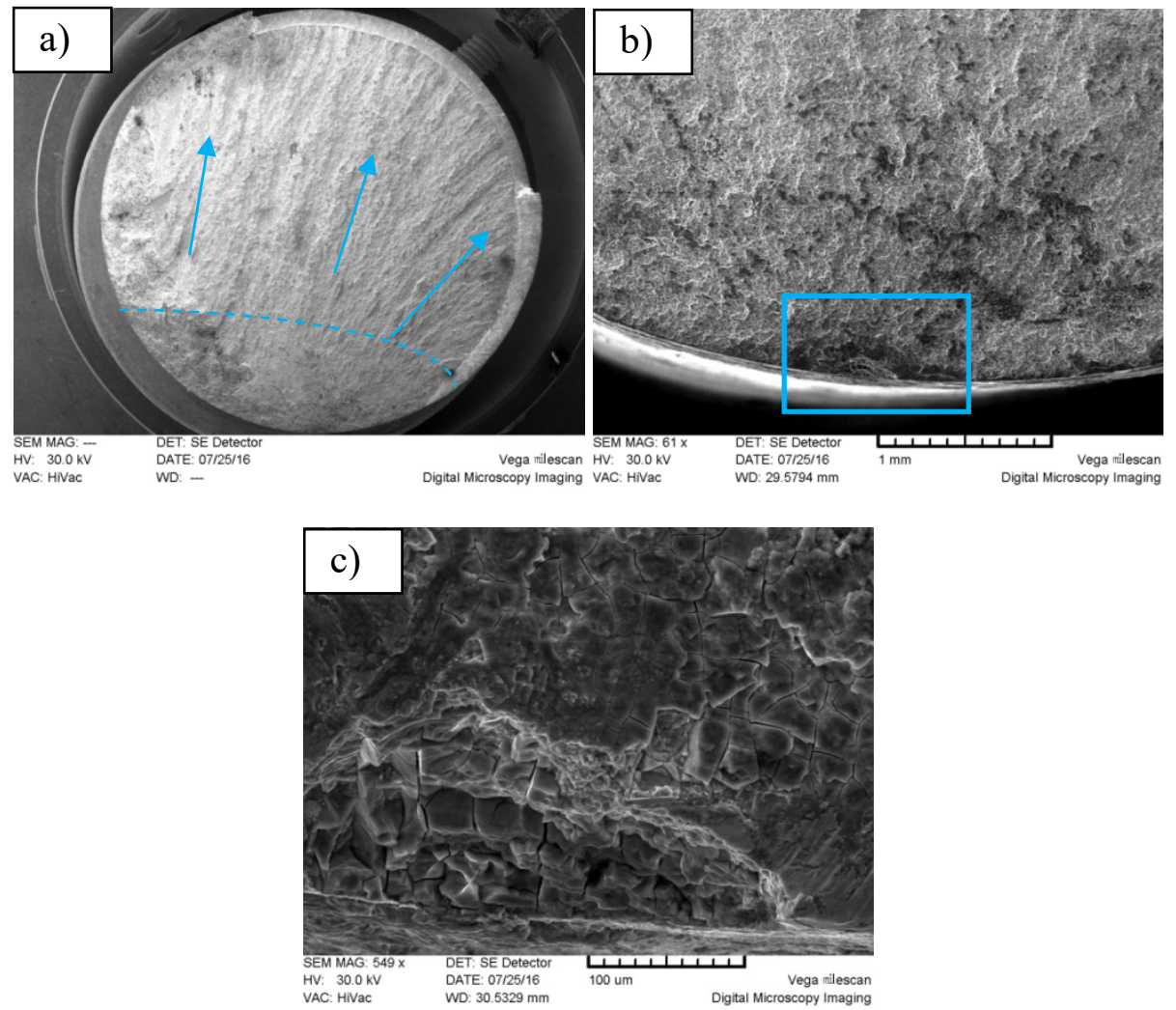

Figure 2: Microstructure of bolt fracture 



Figure 3 Intergranular suger block morphology and secondary crack morphology near fatigue source a)Intergranular cracking in crack growth region; b)Crack near the crack source; c)Energy spectrum curve of crack growth zone

The above characteristics show that the fracture of the bolt is fatigue fracture, the crack originates from the pits on the surface, the expansion area is brittle intergranular cracking, and the area occupied by the crack growth area is small, which is low-cycle fatigue characteristic.

The surface scanning mode of EDX spectrum is used to analysis the law of various elements near the location of fracture, as shown in Figure 4. The Cd content in the crack source region is high (Fig. 4b), and gradually decreases along the crack propagation direction. The $\mathrm{Cr}$ content of source region is high, and also decreases gradually along the direction of crack propagation, and crack area the content of $\mathrm{Cr}$ was higher. The Fe content of crack source region is low, but increases gradually along the direction of crack propagation. The results of energy spectrum scanning show that the fatigue crack originates from the micro-cracks under the $\mathrm{Cd}$ coating, and the $\mathrm{Cr}$ in the micro-crack region is abnormally high. 

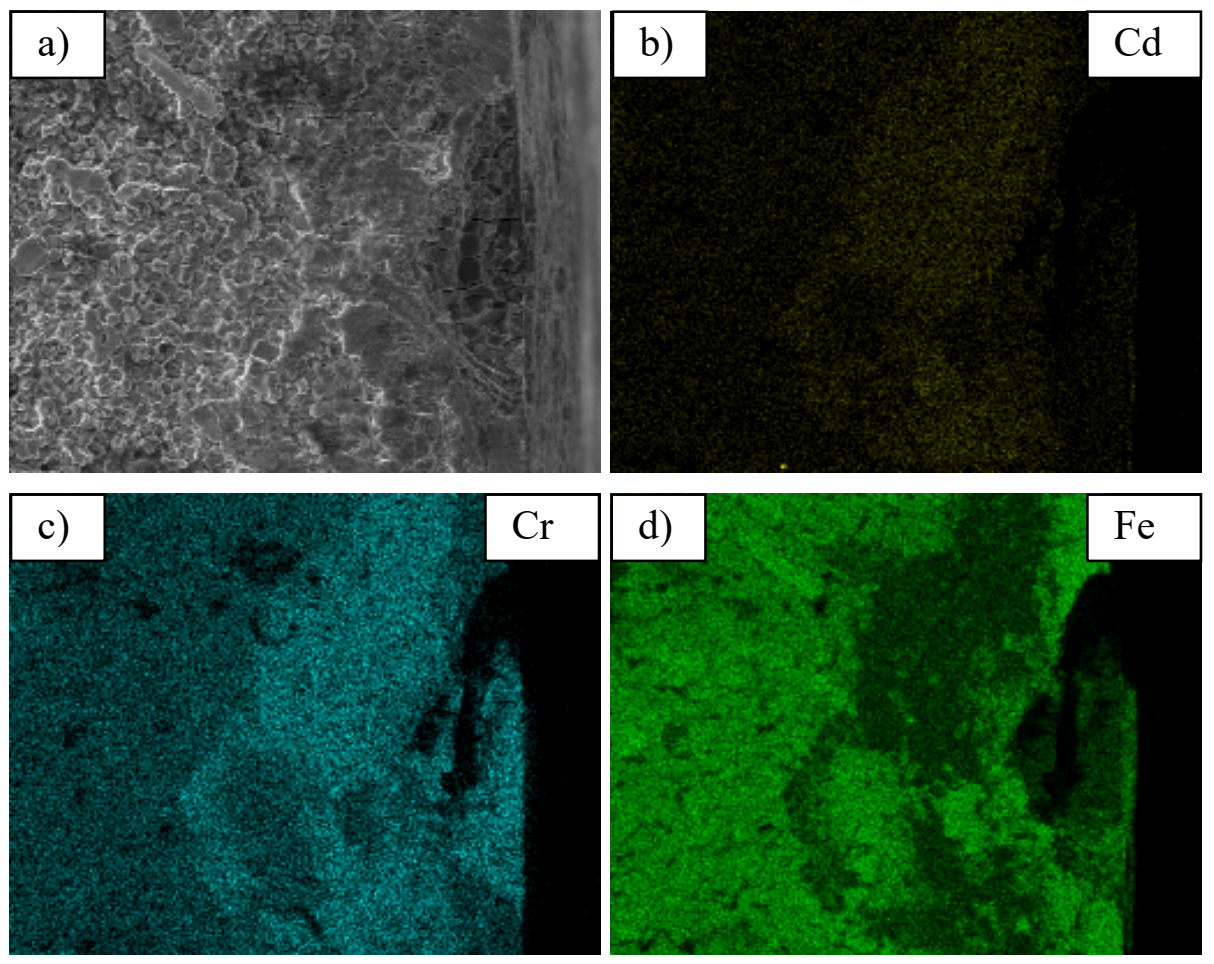

Figure 4: Distribution of chemical elements near fatigue source (upper left is the number of pictures, right is the corresponding chemical elements)

\subsubsection{Micro topography of bolt side wall}

The macroscopic topography of the bolt sidewall is shown in Fig.5. In the vicinity of the crosssection location found a number of pits, the middle position of pit is dark gray, and the surrounding matrix is gray, the colour between the two areas is obvious difference. Finally, one of the pits is located at the location of the bolt fracture section, which is the origin of the fatigue crack. The deposits around the pits were cracked, and micro-cracks were observed in the pits. Cooperation with area-scan function of Energy Distribution Spectrum (EDS), as shown in Fig. 6, the normal region is chrome-plated, and the Cd deposit at the crack region of the dimple area falls off, and the $\mathrm{Cr}$ content in the crack is obviously higher than that in the other places. The distribution of Fe in the matrix is obviously lower than that in the surrounding area.

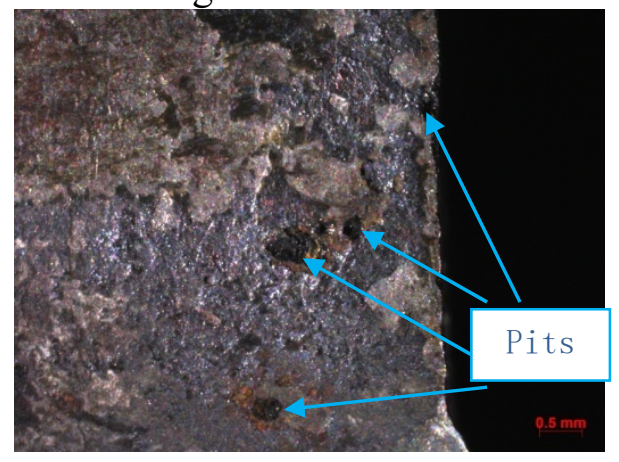

Figure 5: Corrosion pits on the flank of fracture 

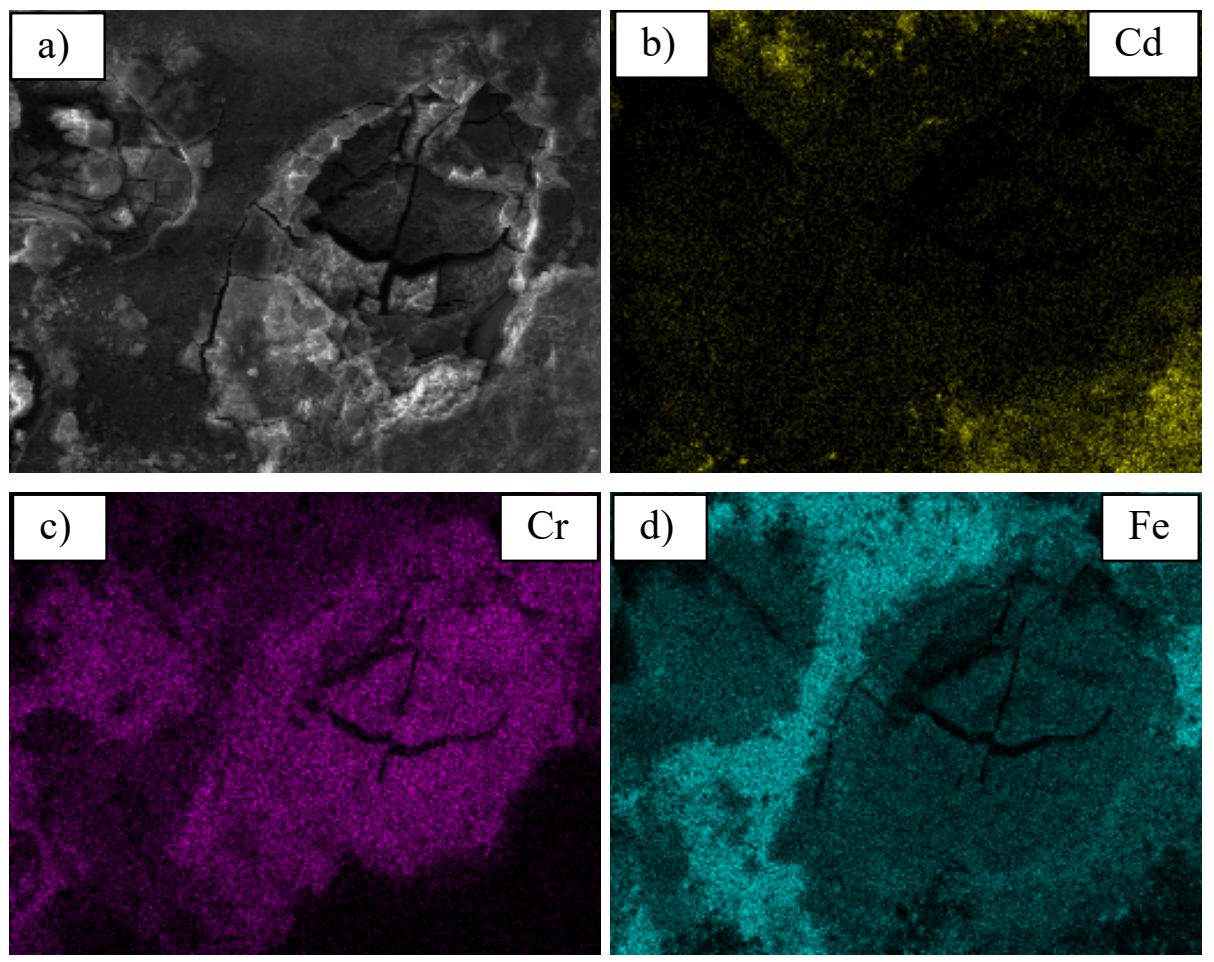

Figure 6: Distribution of chemical elements of corrosion pits (upper left is number of pictures; upper right is corresponding chemical elements)

\section{Discussion}

Based on fracture morphology, the main cause of mainly bolt fracture is related to hydrogen embrittlement bulge in the cadmium plating process. Usually in order to avoid potential corrosion between different metal materials, the bolt surface is cadmium-plated. The energy spectra of the side wall surfaces of the failed bolts indicated that the bolts were indeed cadmium-plated. After cadmium plating treatment, passivation treatment with chromic acid is used to form a chromate passivation layer on the surface of the cadmium layer to improve the anticorrosive ability of the coating. The failure of the bolt surface and the high $\mathrm{Cr}$ content near the crack source may come from the chromate passivation process.

The higher $\mathrm{Cr}$ content in the pits, as seen from the pits on the sidewall surface, indicates that the pits already existed prior to the chromate passivation treatment. A large number of $\mathrm{Cr}$ salt accumulation in the pit. So that, the pits may be formed in the cadmium plating process or its subsequent hydrogen removal process. In the subsequent chromate passivation treatment, chromate deposits cover the surface of the pit, resulting in the defect is not obvious, which passed the NDT test.

A large amount of intercrystalline cracks were found in the fracture zone, and the Cd content in the intergranular crack zone was abnormal. Since the environment and the natural environment did not lead to a large amount of $\mathrm{Cr}$ content, the existing evidence shows that the corrosion pits already exists. During the cadmium process, cadmium or hydrogen into the grain boundary, leading to grain boundary weakening. The crack propagates along the grain boundary at a lower load, and a grain boundary crack is formed. The results show that the cadmium plating process or the subsequent hydrogen removal process may not be suitable, which leading to the existence of hydrogen embrittlement cracks. 


\section{Conclusions}

In summary, the failure of the hub bolt is due to the combined effect of fatigue and hydrogen embrittlement. The crack originates from the pits on the surface of the bolt, and the corrosion pits are the main cause of the origin of the fatigue crack.

The high $\mathrm{Cr}$ content of the etch pits indicates that the corrosion pits are present prior to the chromate passivation process prior to assembly, so the bolt is defective prior to assembly. The content of cadmium in the surface crack growth area is high, and the intergranular crack is also observed. Therefore, the process conditions for the bolt in the process of removing hydrogen after cadmium platingare not appropriate.

\section{Acknowledgements}

The research described in this paper was supported by Natural Science Foundation of China U1633117 and U1533102. The authors acknowledge the financial assistance of the civil aviation science and tehnology fund MHRD20130203.

\section{References}

[1] LIU De-lin, TAO Chun-hu, LIU Chang-kui, JIANG Tao. New Phenomenons and Knowledge of Steel Hydrogen Embrittlement [J]. Failure Analysis and Prevention. 2015 10(6):376-383

[2] Hu C Y,Liu X L,Chen X,et al.Failure analysis of rotating shaft in main undercarriage[J]. Acta Aeronautica et Astronautica Sinica, 2014,35(2):461-468.

[3] CHENG Zong-hui, ZHANG Shi-dong, HUANG Chao, CAO Qiang. Research on Hydrogen Embrittlement Fracture of Fixing Bolt of Undercarriage[J].Failure Analysis and Prevention.2016 11(2):129-132

[4] Sun Xiaoyan, Research on hydrogen embrittlement of bolt[J], Aerospace Standardization,2007(2):1-9

[5] Huang fangqiu. To analysis of the problem in NDT of Boeing-737 wheel hub blots[J].SCIENCE \& TECHNOLOGY INFORMATION, 2013, 7: 78-79

[6] SHAO Xu-fen,HU Cheng-jiang, LUO Yu-shu, LI Ji-tao, REN Shi-gang, HU Jia-jie, DU Nan. Influence of Chromium Electroplating on Fatigue Performance of 300M Steel[J]. Failure Analysis and Prevention. 2012 7(4):129-132

[7]Xu Shiqing. Analysis on hydrogen embrittlement of elastic bush[J]. Electroplating \& Pollution Contiol, 2002,22(5),7-8

[8]Yan Hua, Wen Qingjie, Dai Shucheng. Study of Brush Plating Cadmium Process for High Tensile Strength Steel[J]. Painting \& Electroplating 2007, 4,31-32

[9]Zeng Jiaya. Failure analysis of connecting bolt under Cd coating of engine[J]. JOURNAL OF SOLID ROCKET TECHNOLOGY. 1999, 22(3),71-75 